\title{
Caracterización y análisis comparativo de fórmulas médicas tradicionales utilizadas por sabedores ancestrales del departamento del Chocó, Colombia, para el tratamiento de la diabetes
}

\section{Characterization and comparative analysis of traditional medical formulate used by the department of ancient aware Chocó, Colombia, for the treatment of diabetes}

\author{
Carlos Ariel Rentería Jiménez ${ }^{1}$, Yarleidys Cruz Mosquera², Viky Yency Vega ${ }^{2}$
}

\section{Resumen}

La diabetes es una enfermedad crónica muy común, constituye además un factor de riesgo cardiovascular importantelf, al mismo tiempo uno de los tratamientos más costosos. De alli la importancia de combatirla teniendo diferentes alternativas terapéuticas. En esta perspectiva y conscientes de la importancia de la medicina tradicional, se llevó a cabo un ejercicio de identificación de caracterización de fórmulas médicas tradicionales utilizadas para el tratamiento de la diabetes en zonas rurales de tres municipios del departamento del Chocó (Bagadó, Quibdó, Tadó). A partir de entrevistas a diferentes sabedores ancestrales y miembros de la comunidad, se logró identificar y caracterizar cincofórmulas médicas tradicionales, cuyo análisis comparativo permitió la identificación de 18 plantas medicinales usadas en su preparación (17 géneros y 16 familias botánicas), siendo la familia Myrtaceae la más representativa con 3 especies; 7 especies son de hábito herbáceo, 5 son arbustos, 3 son árboles, 2 corresponden a lianas y 1 hierba epifita. Las plantas medicinales registradas en el presente estudio son en su mayoría silvestres (67\%), otras tanto silvestres como cultivadas (23\%), evidenciándose el hecho que los sabedores ancestrales ubicados en el área de estudio tienen predilección por la flora medicinal que crece de manera silvestre y en su mayoría nativa, de hábito herbáceo, arbóreo y arbustivo, ubicadas sobre todo en paisajes también silvestres, como rastrojos, bosques, orillas de los cuerpos de agua y caminos. Las pruebas fitoquímicas preliminares realizadas a las cinco fórmulas médicas tradicionales revelaron la presencia de compuestos fenólicos, taninos, leucoantocianidinas, cumarinas, flavonoides, leucoantocianidinas y alcaloides, metabolitos secundarios con reconocida actividad antidiabética

Palabras clave: Actividad antidiabética, Compuestos fenólicos, Diabetes mellitus, Fórmulas médicas tradicionales, Medicina tradicional.

\begin{abstract}
Diabetes is a very common chronic disease, and is a major cardiovascular risk factor, while one of the most expensive treatments. Hence the importance combat this disease have different therapeutic alternatives. In this perspective and understand the importance of traditional medicine, it conducted an exercise of identification, characterization of traditional medical formulas used for the treatment of diabetes in rural areas of three municipalities in
\end{abstract}

Investigador Asociado II, Instituto de Investigaciones Ambientales del Pacífico (IIAP), Quibdó, Colombia. e-mail: crenteria@iiap.org.co

2 Contratista Instituto de Investigaciones Ambientales del Pacífico (IIAP), Quibdó, Colombia. e-mail: yacrumo82@hotmail.com e-mail: vikyvega15@gmail.com

Recibido:9 de diciembre de 2013 Aprobado: 19 de febrero de 2014 


\section{Medicina tradicional y diabetes. CA Rentería et al.}

the department of Chocó (Bagadó, Quibdó, Tadó). From interviews with different ancestral knowing and community members, it was possible to identify and characterize five (5) traditional medical formulas, whose comparative analysis allowed the identification of 18 medicinal plants used in its preparation (17 genera and 16 botanical families), it is the most representative with 3 species Myrtaceae family; 7 species are herbaceous habit 5 are shrubs, 3 are trees, lianas and 2 correspond to one epiphyte grass. Medicinal plants recorded in this study are mostly wild (67\%), others are both wild and cultivated (23\%). Demonstrating the fact that the ancestral knowing located in the study area have a predilection for the medicinal flora that grows wild and in its native majority, herbaceous, arboreal and shrubby habit, located mainly in also wild landscapes, as stubble, forests, banks of water bodies and roads. Preliminary tests phytochemical the five traditional medical formulas revealed the presence of phenolic compounds, tannins, leucoantocianidinas; coumarins, flavonoids and alkaloids leucoantocianidinas; secondary metabolites with known antidiabetic activity.

Keywords: Antidiabetic activity, Diabetes mellitus, Phenolics compounds, Traditional medical formulae, Traditional medicine.

\section{Introducción}

La medicina tradicional comprende un sinnúmero de conocimientos y prácticas culturales generados en el seno de una comunidad, especialmente transmitidos de generación en generación, los cuales se basan en un saber ancestral de naturaleza empírica que ofrecen e intentan dar alternativas o soluciones a los problemas de salud o enfermedad de los miembros de la comunidad donde son generados. Debido a que este acervo de conocimientos forma parte de la cultura tradicional, está sujeto a cambios que propician su desarrollo, de tal manera que algunos pueden perderse pero también encontrarse otros con el correr del tiempo.

Actualmente, la medicina tradicional se practica en gran parte en el departamento del Chocó, sobre todo en comunidades rurales, donde en muchos casos constituye la única opción terapéutica para la atención de enfermedades de alto impacto, como la diabetes, una enfermedad cada más frecuente en estas poblaciones.

La diabetes es una enfermedad crónica ocasionada por la dificultad del organismo para metabolizar la glucosa, es decir, para utilizar el azúcar ingerido con los alimentos. Esto determina un aumento del nivel de azúcar en la sangre, o sea, una hiperglucemia. Según la causa que provoca esta alteración, se diferencian dos tipos de diabetes: diabetes mellitus tipo I o insulinodependiente y diabetes mellitus tipo II o no insulinodependiente.

En Colombia, se estima una prevalencia de diabetes para el grupo de edad entre 20 y 79 años en 4,8\% y ajustada por edad en 5,2\%, lo que proporciona una cifra aproximada de un millón y medio de personas con diabetes mellitus tipo 2 y su incidencia sigue en aumento constante; en el año 2020 serán más de 2,5 millones de personas.

La etnobotánica reporta la existencia a nivel mundial de 1000 a 1200 plantas con potencial anti-diabético (Negri 2005, Trojan-Rodríguez et al. 2011). Dentro de la medicina tradicional colombiana se destaca el uso de más de 400 plantas usadas para contrarrestar los síntomas producidos por la diabetes, muchas presentes en el Chocó Biogeográfico colombiano como: hierva de gallina (Agrimonia eupatoria), eucalipto (Eucalyptus globulus), cilantro (Coriandrum sativum), calambombo (Justicia chlorostachya), riñonera (Justicia secunda Vahl), árbol del pan (Artocarpus comunis), amor seco (Bidens pilosa), achiote (Bixa Orellana), capitana (Neorolaena lobata), aguacate (Persea americana Mill), guayaba (Psidium guajava), escobilla (Conobea scoparioides), saúco (Solanum incompetum), verbena (Stachytarpheta callennensis), ruda (Ruta graveolens), zarzamora (Rubus fructicosus), maíz (Zea mays) (García 1974, 1975, Ospina y Pinzón 1995, Rentería-Jiménez et al. 2010, Torres et al. 2013). Muchas de estas plantas no han sido suficientemente estudiadas a fin de conocer sus posibles efectos o beneficios para controlar la glicemia o su aplicación en virtud de su eficacia y seguridad (Volpato et al. 2002).

Plantas medicinales que son indicadas principalmente por sabedores ancestrales (personas con muchos años de experiencia en la cura y/o alivio de afecciones o enfermedades en comunidades negras del departamento del Chocó) a través de diferentes nombres comunes o tradicionales, lo que genera dificultades para su estudio por las constantes variaciones que tienen estos nombres de una región a otra, dificultando de esta forma su estudio (Rentería-Jiménez et al. 2010). A esto se suma que muchos de los tratamientos propuestos por los médicos tradicionales constituyen una combinación de varios extractos de plantas, que probablemente sea necesaria para que se dé el efecto biológico deseado (sinergismo).

Esta investigación presenta los principales resultados obtenidos por investigadores del Instituto de Investigaciones Ambientales del Pacífico (IIAP) durante los últimos cuatro años, derivados a partir de la realización de entrevistas y conversaciones informales con médicos tradicionales del departamento del Chocó, Colombia, que permitieron la caracterización de diferentes fórmulas médicas tradicionales usadas para aliviar los síntomas relacionados con la diabetes y realizar un análisis comparativo entre varias de estas fórmulas a partir de información relacionada con 


\section{Bioetnia Volumen 11, 2014}

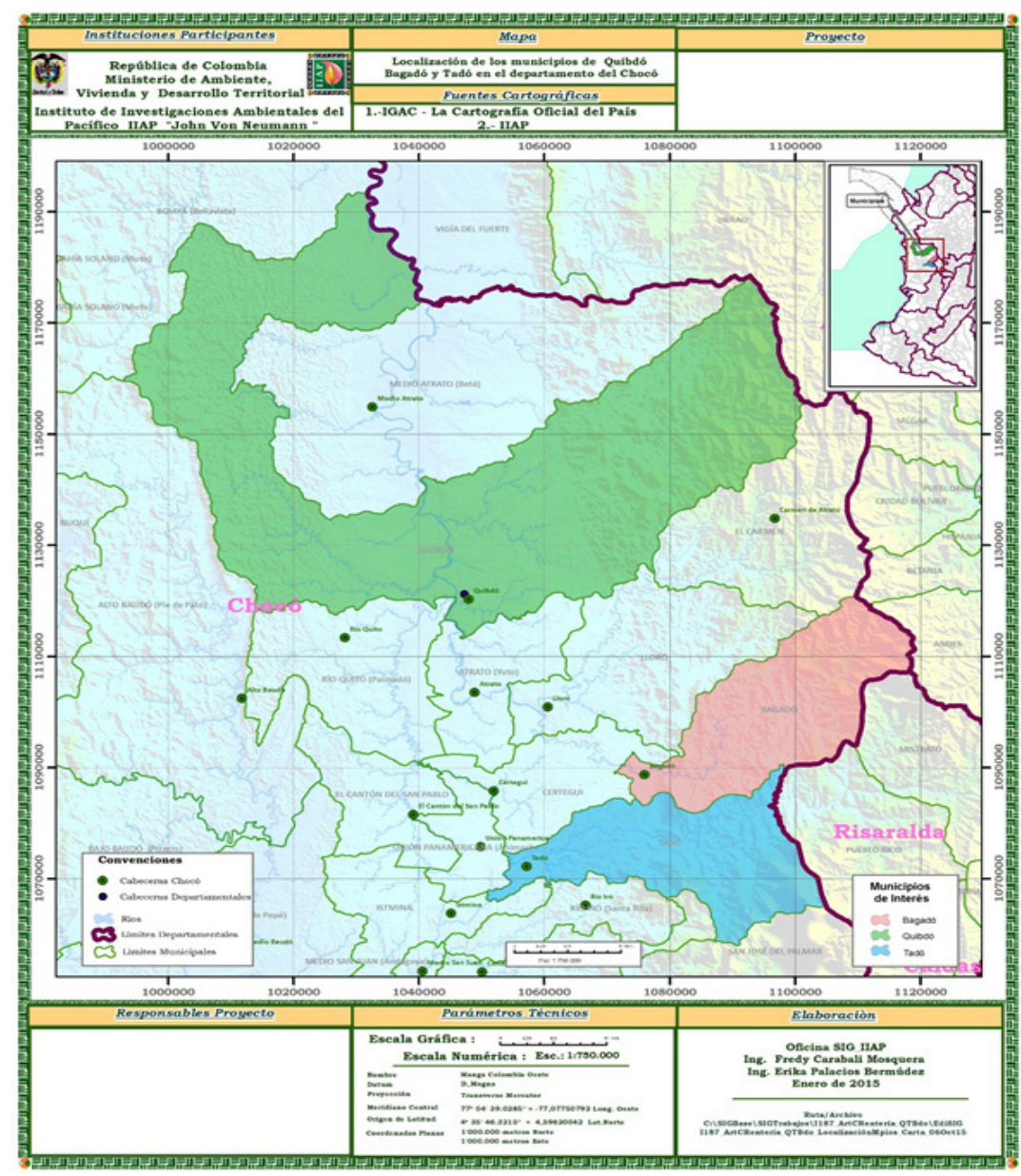

Figura 1. Ubicación geográfica del área de estudio, municipios de Quibdó, Tadó y Bagadó.

las principales características taxonómicas de las plantas empleadas, partes usadas, formas de preparación, modos de empleo, dosificaciones y contraindicaciones.

\section{Metodología}

Area de estudio. El estudio se desarrolló en tres localidades (Quibdó, Bagadó y Tadó) todas pertenecientes al departamento del Chocó, Colombia (Figura 1).

Quibdó. El municipio de Quibdó se encuentra ubicado en la región natural de la costa pacífica a $43 \mathrm{msnm}$ y sobre la margen derecha del río Atrato; astronómicamente se encuentra a los $5^{\circ} 40^{\prime}$ norte y $76^{\circ} 40^{\prime}$ oeste, su extensión total es de $6164 \mathrm{~km}^{2}$, con una temperatura de $28^{\circ} \mathrm{C}$ y una precipitación de $10.749 \mathrm{~mm}$ anuales.

Bagadó. Ubicado a la margen izquierda del río Andágueda, a $05^{\circ} 24^{\prime} 32^{\prime \prime}$ de latitud norte y $76^{\circ} 25^{\prime} 16^{\prime \prime}$ de longitud oeste, a una altura sobre el nivel del mar de $200 \mathrm{msnm}$, con una temperatura media de $28^{\circ} \mathrm{C}$ y una precipitación media anual de $7.800 \mathrm{~mm}$. Dista de Quibdó $80 \mathrm{~km}$ (Arango y Saldarriaga 1997).
Tadó. El municipio de Tadó está ubicado en la parte oriental del departamento del Chocó, dentro de la zona del Alto San Juan, con un área aproximada de $878 \mathrm{~km}^{2}$, su cabecera municipal se localiza a los $76^{\circ} 73^{\prime} 10^{\prime \prime} \mathrm{W}$, al margen izquierdo del río San Juan. Localizado a $75 \mathrm{msnm}$, con una temperatura promedio entre $27^{\circ} \mathrm{C}$ y $28^{\circ} \mathrm{C}$ (EOT, Alcaldía Municipal de Tadó 2009).

\section{Métodos}

Identificación de sabedores ancestrales. A través de la aplicación de entrevistas y charlas informales a miembros de las comunidades asentados en el área de estudio, quienes a partir de su experiencia como pacientes o usuarios de la medicina tradicional ayudaron en la identificación de sabedores ancestrales con especial énfasis en los que ejecutan tratamientos dirigidos a la cura de la diabetes. Cada sabedor ancestral fue entrevistado a fin de obtener información de primera mano en relación con el origen de su conocimiento y años de experiencia en el ejercicio de la medicina tradicional 


\section{Medicina tradicional y diabetes. CA Rentería et al.}

Plantas medicinales presentes en las fórmulas médicas tradicionales. Para este aspecto en particulary entendiendo el proceso progresivo en el que se desarrolló el estudio, se procedió a realizar colectas y tomar el registro de las plantas utilizadas por los sabedores ancestrales para el tratamiento de la diabetes, a fin de realizar su identificación taxonómica. El material botánico fue identificado en el Herbario de la Universidad Tecnológica del Chocó, en algunos casos partiendo de su nombre vulgar y haciendo la respectiva verificación de la muestra y en otros haciendo comparación directa de las muestras con el material herbolario concentrado en esta colección botánica del Chocó Biogeográfico.

Caracterización de fórmulas médicas tradicionales. Se pidió a cada sabedor ancestral que preparara la fórmula usada para el tratamiento de la diabetes y de esta forma se registraron datos como las partes y cantidades de cada planta usada, formas de preparación y duración de cada tratamiento.

\section{Resultados y discusión}

Sabedores ancestrales identificados. Se identificaron un total de cinco sabedores ancestrales, todos hombres, con edades entre 55 y 73 años, con un gran reconocimiento social y amplia experiencia (entre 40 y 50 años) en la aplicación del tratamiento para la cura y/o alivio de la diabetes (Tabla 1). El promedio de edad de estos sabedores confirma lo encontrado en otros estudios, en el sentido que este conocimiento basado en el empleo de plantas medicinales, se está quedando en las personas mayores y corre el riesgo de perderse como consecuencia de la poca transmisión oral debido a la apatía de las nuevas generaciones, celos en el manejo de la información, entre otros factores (IIAP 2012,
Yates y Ramírez-Sosa 2004).

En relación con las respuestas dadas por los sabedores en cuanto al origen de los conocimientos relacionados con el uso de plantas medicinales (Tabla 1), confirman que estos conocimientos son aprendidos de sus ancestros (abuelos, padres, tíos, entre otras), además del intercambio de información con otros médicos tradicionales, posibilitando el diálogo de saberes y permite seguir avanzando y retroalimentando sus conocimientos día a día.

Plantas medicinales presentes en las diferentes fórmulas médicas tradicionales. Se identificaron un total de cinco fórmulas médicas tradicionales usadas para contrarrestar los síntomas producidos por la diabetes, las cuales utilizan en su preparación 18 especies de plantas medicinales pertenecientes a 18 géneros y a 16 familias botánicas, siendo la familia Myrtaceae con 3 especies la más comúnmente reportada; del resto de familias usa una sola especie: Fabacea, Lauracea, Costaceae, Moraceae, Urticaceae, Gesneriaceae, Bignobaceae, Dilleniaceae, Clusiaceae, Cucurbitaceae, Equisetaceae, Smilacaceae, Rutaceae, Cosmelinaceae, además de un helecho o Pteridofito, perteneciente a la familia Dennstaedtiaceae (Tabla 2). Resultados que solo coinciden en la familia Fabaceae con los reportados por Marles y Farnsworth (1995), quienes destacan como las familias botánicas que contribuyen con más especies antidiabéticas en todos los países además de la Fabaceae son las Asteraceae, Lamiaceae, Liliaceae, Poaceae y Euphorbiaceae.

La mayoría de las plantas analizadas corresponden a hierbas (7), seguida de arbustos (5) y árboles (3); se registraron (2) lianas y (1) hierba epifita. En cuanto a su origen, 10 especies son nativas, 4 introducidas y 4 naturalizadas. Las plantas medicinales registradas en el presente estudio son

Tabla 1

Sabedores ancestrales entrevistados

\begin{tabular}{lclc}
\hline Médico tradicional & Edad (años) & Origen del conocimiento & Experiencia (años) \\
\hline Américo Agualimpia & 55 & Aprendió de sus padres & 26 \\
Ángel Yanes & 58 & aprendió este arte de sus padres & 30 \\
Antonio Valoyes & 60 & Aprendió de sus ancestros & 35 \\
Manuel Arcindo García Martínez & 56 & Aprendió de sus padres: & 20 \\
& & Pablo García conocido como el & \\
Hugo Crescenciano Lozano & 73 & Mago & Aprendió de sus ancestros, también \\
& \multicolumn{4}{c}{$\begin{array}{l}\text { Con los indios y de los señores } \\
\text { Mena, Trinidad Moreno y Juan }\end{array}$} \\
\end{tabular}


Bioetnia Volumen 11, 2014

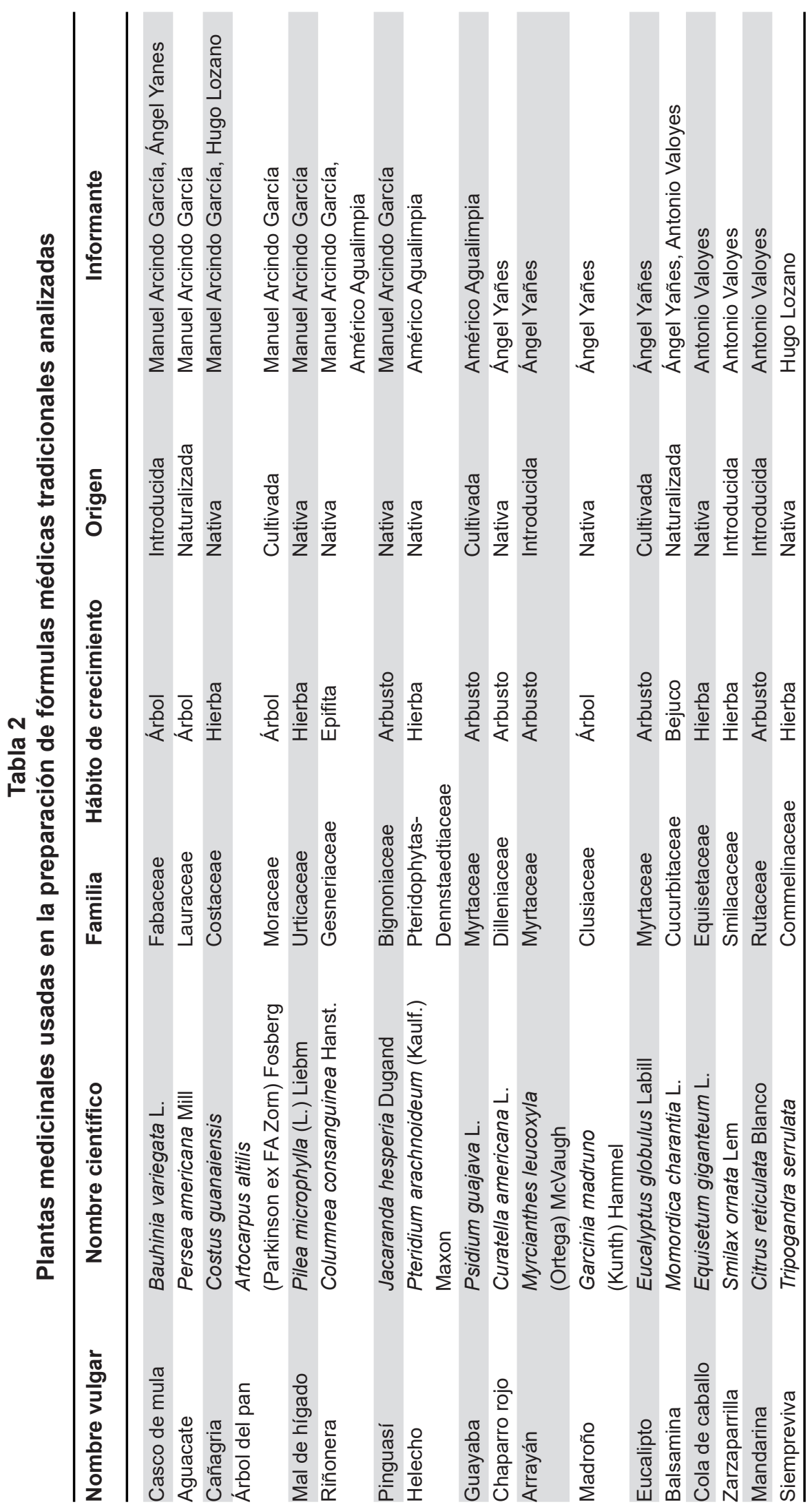

en su mayoría silvestres (67\%), otras son tanto silvestres como cultivadas (23\%), evidenciándose el hecho de que en el área de estudio se emplean sobre todo plantas exclusivamente silvestres (67\%), hallazgo similar al obtenido por Zuluaga (2003), quien refiere que los sabedores ancestrales afrocolombianos del río Anchicayá, Valle del Cauca, Colombia, tienen predilección por la flora medicinal que crece de manera silvestre $(66 \%)$. De igual manera y en relación con el estudio realizado por Zuluaga (2003) con comunidades afrocolombianas del Pacífico, se evidencia como los afrodescendientes han logrado apropiarse de una vegetación de predominio silvestre y en su mayoría nativa, de hábito herbáceo, arbóreo y arbustivo, ubicada en paisajes también silvestres, como rastrojos, bosques, orillas de los cuerpos de agua y caminos.

En relación con las especies más usadas en la preparación de las fórmulas médicas, se destaca el uso de cuatro especies. La primera, es una especie comúnmente conocida como casco de mula (Bauhinia variegata) debido a la forma de sus hojas, actualmente aprobada en el vademécum colombiano de plantas medicinales (Ministerio de la Protección Social 2008) como hipoglicemiante, en la medicina popular; las hojas se utilizan en infusiones para tratar la diabetes (Hoyos 1992). La segunda especie es el chaparro rojo (Curatella americana) también aprobado en el vademécum, se utiliza para disminuir el azúcar en la sangre, para lo cual se recomienda la toma de dos tazas diarias de la decocción de la corteza. Echeverry (1984) (citado en Bernal y Correa 1990 ) manifiesta que la 


\section{Medicina tradicional y diabetes. CA Rentería et al.}

madera rojiza de algunas variedades de este árbol se vende en las plazas de mercado colombianas como antidiabéticos. Piñeros et al. 1991 (citados en Bernal y Correa, 1990) recomiendan por su efectividad, el uso de la corteza en decocción para la diabetes, tomado dos tazas diarias. Las últimas dos especies son conocidas como cañagria (Costus guanaiensis) y riñonera (Columnea consanguínea.

Caracterización de las fórmulas médicas tradicionales. Las cinco fórmulas médicas tradicionales identificadas fueron caracterizadas, obteniéndose información detallada en relación con sus diferentes formas de preparación, dosificación y contraindicaciones; se registraron además datos como las partes y cantidades de cada planta usada y formas de preparación (Tabla 3).

En cuanto a la forma de preparación de las fórmulas, se encontró que todos los sabedores ancestrales utilizan la misma técnica de preparación(decocción) por espacios de 45 minutos en promedio, que luego dejan enfriar por un tiempo de 10 a 20 minutos, luego son envasadas y almacenadas preferiblemente en refrigeración para ser suministradas a los pacientes, pero difieren en las porciones de las plantas y las especies usadas (Tabla 3 ).

En relación con la forma de administración, se destaca el uso de la vía oral que de acuerdo con las entrevistas realizadas a los médicos tradicionales, manifiestan que los tratamientos tradicionales surten mejores resultados cuando son suministrados por vía oral a los pacientes, debido a que las plantas medicinales pueden realizar su proceso de forma más rápida al tener contacto directo con los órganos internos, teniendo así más efectividad.

Se concluye además, que la dosificación más común es dos vasos diarios, de aproximadamente $250 \mathrm{ml}$, uno al levantarse y otro al acostarse. $\mathrm{Y}$ en relación con las contraindicaciones las más comunes son las de no comer alimentos dulces, ni tomar bebidas alcohólicas, debido a que no le permitiría tener un buen estado de salud y a la vez puede causarle afecciones más críticas llevándolos hasta la muerte.

Con respecto a las partes de la planta más utilizadas en la preparación de las fórmulas, se observa que el mayor uso lo tienen las hojas (53\%), seguido de la planta entera $(16 \%)$, el tallo (11\%), y finalmente cogollos y cáscaras con (10\%) respectivamente.

De la evaluación fitoquímica preliminar. Los resultados del estudio fitoquímico cualitativo, de los extractos acuosos de las cinco composiciones estudiadas, se presenta en la Tabla 4. Vemos que en todas las composiciones predominan los compuestos fenólicos, los taninos y las leucoantocianidinas; en la composición dos, predominan los compuestos fenólicos, taninos, cumarinas, flavonoides, leucoantocianidinas y alcaloides.

La presencia de estos metabolitos secundarios en las composiciones etnomedicinales analizadas coincide de manera particular con la naturaleza química de los compuestos hipoglucemiantes que más frecuentemente se han aislado de plantas con reconocida actividad antidiabética tales como: alcaloides, glucopéptidos, terpenoides, flavonoides, esteroides y compuestos de naturaleza lipídica los cuales son mencionados en los resultados de marchas fitoquímicas que ofrecen algunos estudios de hipoglicemia (Marles y Farnsworth 1995, Castañeda et al. 2004a, 2004b). Esto permite generar mayor evidencia a favor del uso de fórmulas médicas tradicionales derivadas de la combinación de diferentes plantas medicinales en estudio para el tratamiento de la diabetes. Abrão de Oliveira (2010), Arroyo et al. (2009) y Simonovska et al. (2003) concuerdan en que los flavonoides y compuestos fenólicos tienen estrecha relación con la actividad antioxidante, lo que ha sido reportado como muy significativo en la prevención de la diabetes y como se muestra en los resultados, las cinco fórmulas tradicionales en estudio contienen estos metabolitos.

Es pertinente recordar que el tipo 2 de diabetes, que afecta a mayor cantidad de personas (OMS 2012), se genera por una incapacidad de las membranas celulares, de los órganos diana, para reconocer la insulina debido precisamente al daño causado por los radicales libres ( $\mathrm{Si}$ monovska et al. 2003). El consumo de infusiones de estas cinco fórmulas tradicionales, y por ende, de antioxidantes presentes en forma de flavonoides, compuestos fenólicos y alcaloides, ayudaría a la protección de las membranas, lo que redundaría en una mejora de la salud.

\section{Conclusión}

Gracias a la enorme biodiversidad vegetal presente en el departamento del Chocó, se observa en diferentes estudios etnobotánicos que es relativamente amplio el número de especies vegetales usadas con fines medicinales basados en conocimientos culturales tradicionales y empíricos. Esta amplia y variada utilización indica la alta experimentación por parte de comunidades ancestrales, asociada posiblemente con el arraigo hacia sus territorios. A pesar de la influencia de la medicina occidental y de la fragmentación de las comunidades ancestrales, como aspecto positivo se observa que los miembros de las comunidades negras comparten sus conocimientos y transmiten estos de una generación a otra.

Adicionalmente, en varios casos en las observaciones hechas en el trabajo de campo también se nota que hay diferencias en diversas localidades en la parte usada de la planta, la posología, la forma de preparación y el uso de coadyuvantes. Incluso plantas muy usadas en una localidad ni siquiera son conocidas en otras, a pesar de ser regiones cercanas o tener un ancestro cultural común. Sin embargo, lo 
Bioetnia Volumen 11, 2014

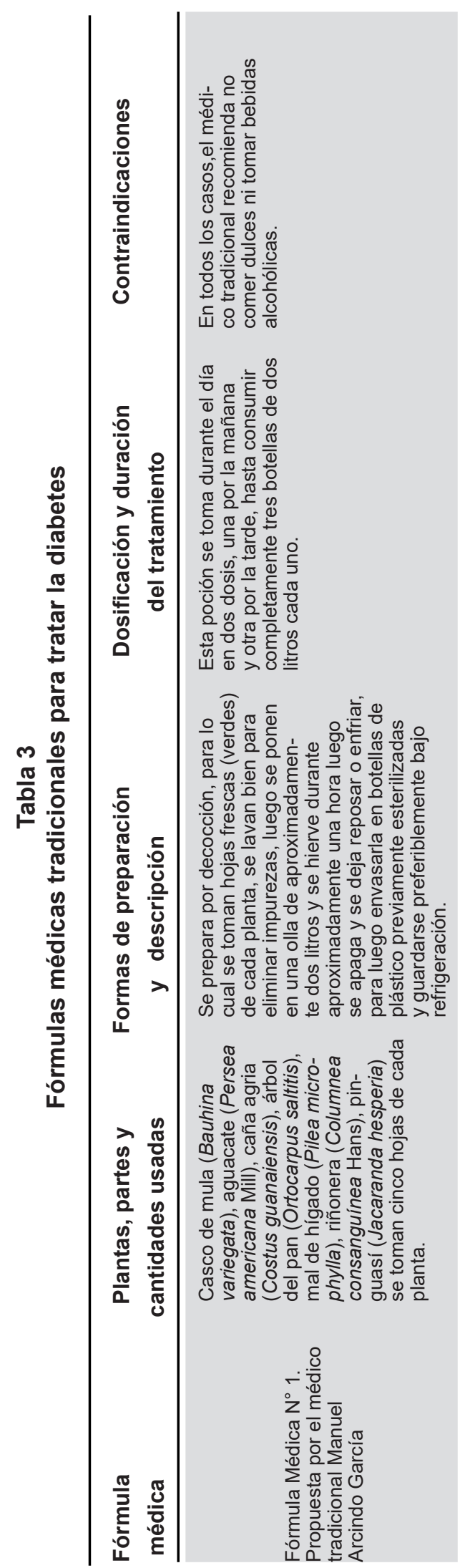

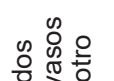

है

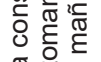

需要

든 응

\%

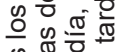

융 $\frac{\pi}{0} \frac{\pi}{0}$

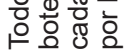

\section{क्ष}

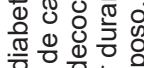

주일

응응

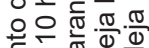

ब

है⿴囗十心

సٓ일

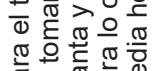

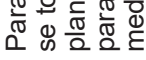

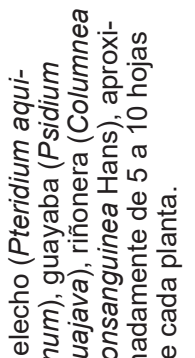

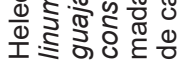

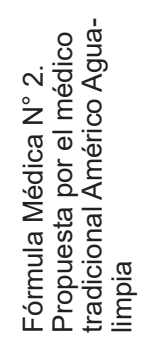

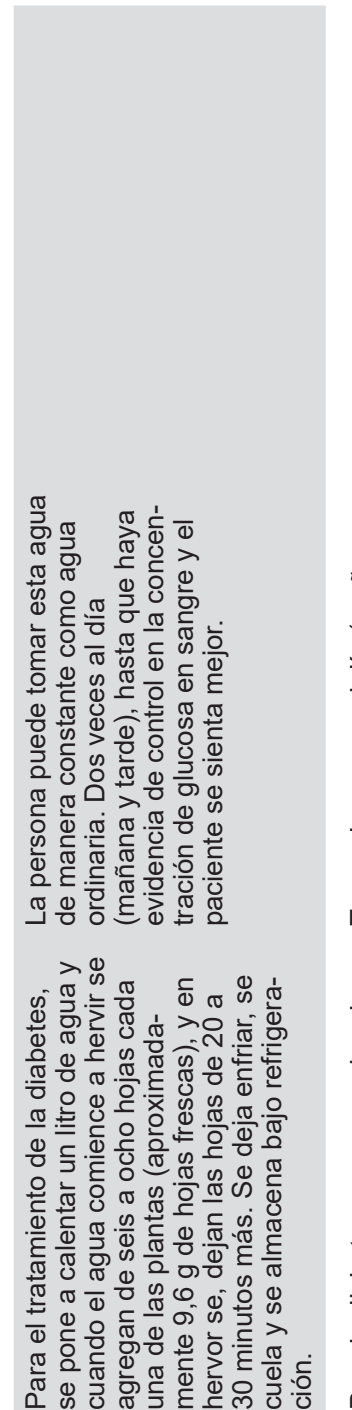

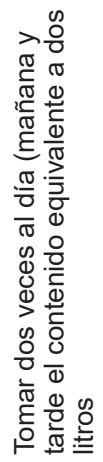

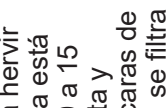

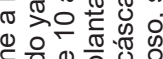

능 융

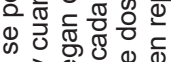

के

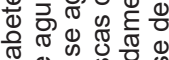

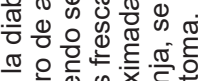

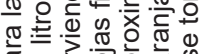

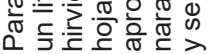

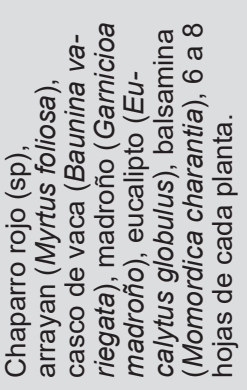

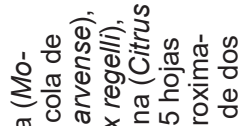

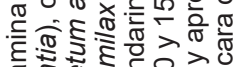

ह ส बं

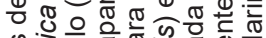

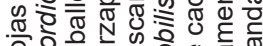

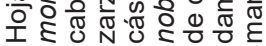

용 \&

융

$>\bar{\varepsilon}$ क

동요

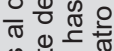

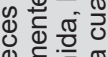

$\Phi$

क वृ 웡

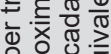

힝ㅎㅇ 항

क) $\frac{\pi}{0} \frac{\pi}{0} \frac{\pi}{0}=$

융응 \&

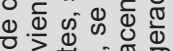

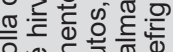

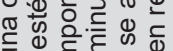

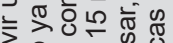

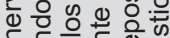

ส

¿ 0 윰

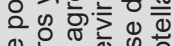

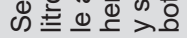

홍으

क्षेत

बृ $\frac{8}{8}$

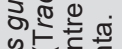

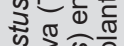

ن $>0$

증 응

ठซ. है है

त)

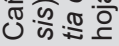
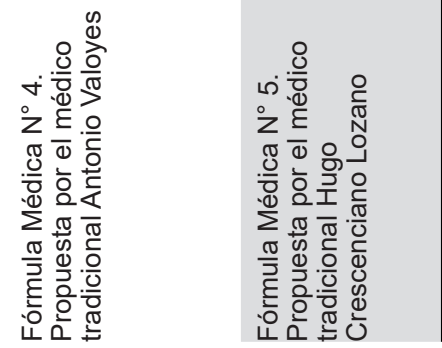


\section{Medicina tradicional y diabetes. CA Rentería et al.}

Tabla 4

Análisis fitoquímico preliminar de las cinco fórmulas médicas tradicionales analizadas

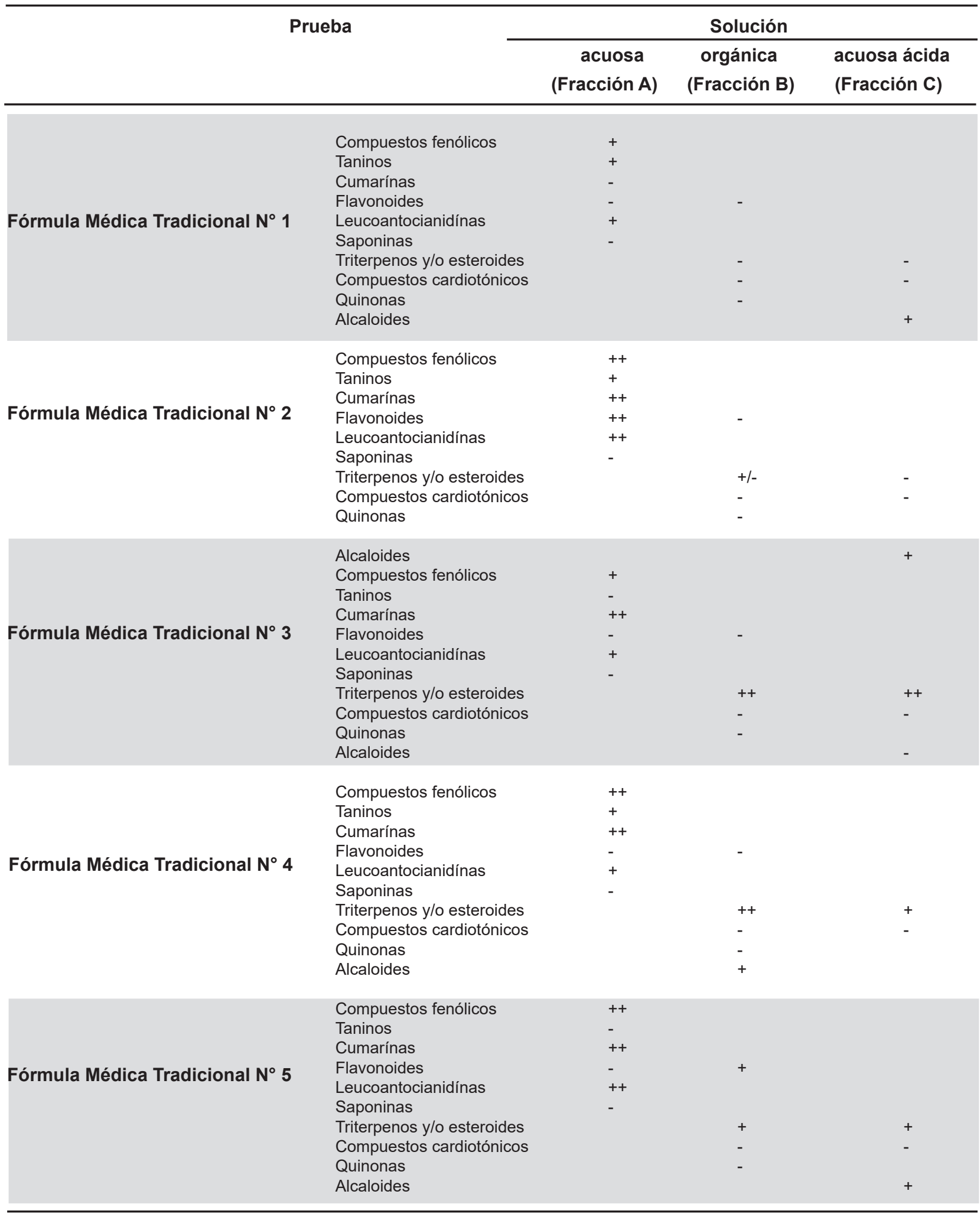




\section{Bioetnia Volumen 11, 2014}

más frecuente que se encontró en los estudios etnobotánicos realizados es que haya coincidencia en especies usadas y en muchas indicaciones, lo que se podría explicar por la poca influencia de otras culturas.

Aun cuando los resultados de este estudio son preliminares, en este trabajo se registra un importante número de especies de plantas medicinales usadas para el tratamiento de la diabetes, algunas de ellas con propiedades fitoquímicas y farmacológicas corroboradas en laboratorio.

Se observa en la muestra de fórmulas médicas tradicionales analizadas diversidad de familias botánicas, no obstante, prevalecen especies pertenecientes a las Fabaceae, Asteraceae, Lamiaceae, Liliaceae, Poaceae y Euphorbaceae, lo que permite inferir la importancia de estas familias en la biodiversidad botánica de la región.

Del análisis de las partes usadas se desprende que las hojas ocupan el primer lugar con respecto al órgano vegetal de mayor importancia terapéutica para control de la diabetes, seguido de la planta entera, tallos y cogollos y cáscaras, tal vez, ello es debido a la facilidad que presentan los órganos blandos para su utilización a través de técnicas sencillas que no implican mayores tecnologías ni inversión en energía calórica.

\section{Literatura citada}

Abrão de Oliveira L. 2010. Yacon (Smallanthus sonchifolius): Compostos fenólicos totais e efeitos sobre a glicemia e estresse oxidativo em ratos diabéticos. Dissertação (Mestrado). Universidade Estadual Paulista "Julio De Mesquita Filho", Faculdade de Ciências Farmacêuticas, São Paulo, Brasil.

Alcaldía Municipal de Quibdó. 2003. Plan de Ordenamiento Territorial de Quibdó. Alcaldía Municipal de Quibdó. 117 pp.

Alcaldía Municipal de Tadó. 2009. Esquema de Ordenamiento Territorial de Tadó. Alcaldía Municipal de Tadó. 117 pp.

Arango S, Saldarriaga MT. 1997. Monografia del Chocó. Quibdó: Editorial de Autores Chocoanos.

Arroyo J, Martínez J, Ronceros G, Palomino R, Villarreal A, Bonilla P, et al. 2009. Efecto hipoglicemiante coadyuvante del extracto etanólico de hojas de Annona muricata L (guanábana), en pacientes con diabetes tipo 2 bajo tratamiento de glibenclamida. An Fac Med. 70 (3): 163-7.

Bernal HY, Correa JE. 1990. Especies vegetales promisiorias de los paises del Convenio Andrés Bello. Instituto Andrés Bello: Bogotá.

Barbosa-Castillo CE. 1992. Contribución al conocimiento de la florula del Parque Nacional Natural El Tuparro. Serie Biblioteca Andrés Posada Arango, libro 3. Bogotá: Instituto Nacional de los Recursos Naturales Renovables y del Ambiente (INDERENA); 271 pp.

Castañeda CB, Manrique RM, Ibañez LV. 2004a. Estudio fitoquímico y farmacológico de plantas con efecto hipoglicemiante. Cultura. 22 (18): 33-59.

Castañeda CB, Manrique RM, Ibañez LV. (2004b). Efecto hipoglicemiante y sobre la lipidemia de Notholaena nivea, "Cuti-Cuti". Revista Horizonte Médico. 4 (1): 2-22.

García BH. 1974-1975. Flora medicinal de Colombia. Bogotá: Imprenta Nacional; pp. 79-87.

González F, Díaz J, Lowy P. 1995. Flora ilustrada de San Andrés y Providencia con énfasis en plantas útiles. Bogotá: Universidad Nacional de Colombia, SENA, Instituto de Ciencias Naturales; pp. 285.

Hoyos J. 1992. Arboles tropicales ornamentales cultivados en Venezuela. Caracas: Sociedad de Ciencias Naturales La Salle; 272 pp.

Mahecha G, Ovalle A, Camelo D, Rozo A, Barrero D. 2004. Vegetación del territorio CAR. 450 especies de sus llanuras y montañas. Bogotá: Corporación Autónoma Regional de Cundinamarca (CAR): 871 pp

Marles RJ, Farnsworth NR. 1995. Antidiabetic plants and their active constituents. Phytomedicine. 2 (2): 137-89.

Menéndez Valderrey JL. "Eucalyptus globulus labill” Asturnatura.com 113. [consultado en octubre 25 de 2014]. Disponible en: www.asturnatura. com/especie/eucaliptus-globulus.html

Ministerio de la Protección Social. 2008. Resolución $N^{\circ} 0425$ de febrero de 2008. Bogotá: MinProtección Social.

Negri G. 2005. Diabetes melito: plantas e princípios ativos naturais hipoglicemiantes. Rev Bras Ciên Farmac. 41: 121-40.

Organización Mundial de la Salud (OMS). 2012. Diabetes. Nota descriptiva $N^{\circ} 312$. [Página Web en Internet]. [fecha de acceso 24 de febrero de 2014]. Disponible en: http:/www.who.int/mediacentre/factsheets/fs312/es/

Ospina LF, Pinzón R. 1995. Plantas usadas como antidiabéticas en la medicina popular colombiana. Rev Colomb Cienc Quim-Farmac. 23: 81-94.

Pino-Benítez CN. (2006). Botánica y screening fitoquímico de doce plantas usadas en medicina tradicional en el departamento del Chocó, Colombia. Rev Latinoam Recur Nat. 2 (1): 33-44.

Rentería-Jiménez CA, Lozano H, García M. 2010. Validación de tratamientos basados en medicina tradicional, dirigidos a la cura de enfermedades de alta ocurrencia nacional y mundial. Hacia la obtención de patentes etnofarmacológicas. Informe final de investigación. Quibdó: Instituto de Investigaciones Ambientales del Pacífico.

Rodríguez JO, Peña JR, Plata E. 1984. Flora de los Andes. Cien especies del Altiplano Cundi-Boyacense. Bogotá: Corporación Autónoma Regional de Cundinamarca (CAR); pp. 150-5.

Simonovska B, Vovk I, Andrenšek S, Valentová K, Ulrichová J. 2003. Investigation of phenolic acids in yacon (Smallanthus sonchifolius) leaves and tubers. J Chromatograp A. 1016 (1): 89-98.

Trojan-Rodríguez M, Alves TLS, Soares GLG, Ritter MR. 2011. Plants used as antidiabetics in popular medicine in Rio Grande do Sul, southern Brazil. J Ethnopharmacol 139 (1): 155-63.

Torres F, Paz G, Zapata M. 2013. Las plantas pueden ser fuente de compuestos antidiabéticos que aún no han sido científicamente validados. Ciencia \& Salud. 1 (3): 11-8.

VanAcker SA, Van Den Berg DJ, Tromp MN, Griffioen DH, Van BennekomWP, Van Der Vijgh WJ, et al. 1996. Structural aspects of antioxidant activity of flavonoids. Free Radical Biol Med. 20 (3): 331-42.

Volpato GT, Damasceno DC, Calderón IMP, Rudge MVC. 2002. Revisão de plantas brasileiras com comprovado efeito hipoglicemiante no controle do diabete mellitus. Rev Bras Plant Med. 4: 35-45.

Yates S, Ramírez RC. 2004. Ethnobotanical knowledge of Brosimum alicastrum (Moraceae) among urban and rural El Salvadorian adolescents. Econ Bot. 58 (1): $72-7$.

Zuluaga G. 2003. La botella curada: un estudio de los sistemas tradicionales de salud en las comunidades afrocolombianas del Chocó Biogeográfico. Bogotá: Amazon Conservation Team, Instituto de Etnobiología, Universidad del Bosque. 352 pp. 


\section{Medicina tradicional y diabetes. CA Rentería et al.}

\section{Anexo 1 \\ Descripción de las 18 plantas medicinales identificadas}

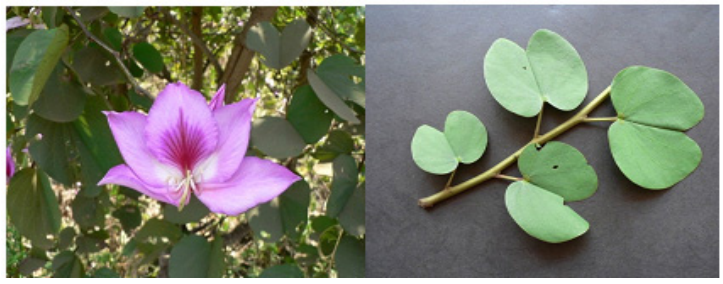

Figura 2. Casco de mula (Bauhinia variegata). Árbol de 10 a $12 \mathrm{~m}$ de altura, tronco corto y erecto; corteza de color gris marrón y hendida verticalmente. Hojas de 5 a $15 \mathrm{~cm}$ de largo, profundamente lobuladas, presentan de 11 a 13 nervios que salen desde la base, pubérculos en los nervios de la cara inferior y base truncada hasta profundamente cordiforme. Flores conspicuas, de color morado, rosa brillante o blanco, bastante vistosas, fragantes, de 8 a $12 \mathrm{~cm}$ de diámetro, con cinco pétalos. Fruto en forma de vaina o legumbre aplanada de 15 a $30 \mathrm{~cm}$ de longitud, su dehiscencia se abre desde el ápice hacia la base y contiene varias semillas de color marrón claro y miden $1 \mathrm{~cm}$ de diámetro (Hoyos 1992). Presencia de varios compuestos aislados e identificados, incluyendo lactonas, flavonoides, triterpenos, glicósidos, esteroles, taninos, alcaloides, saponinas, por lo cual, ellos o uno de ellos sea el responsable del efecto terapeútico y genotóxico en el organismo.

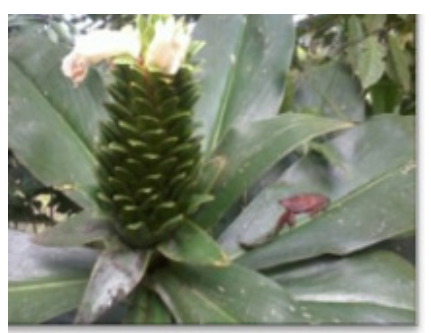

Figura 3. Cañagria (Costus aff. guanaiensis Rusby). Hierba arbustiva, musoide, suculenta; pseudotallo rojizo, $4 \mathrm{~m}$ de altura. Hojas alternas, equidistantes, ovaladas, $50 \mathrm{~cm}$ de longitud, $8 \mathrm{~cm}$ de ancho en la parte media, pecíolo transformada en vaina que envuelve el tallo. Inflorescencias terminales, flores blancas con parche amarillo y ápice rojizo; brácteas verdes en el ápice, rojizas en la superficie externa y vino tinto en la superficie interna; cáliz vino tinto, pétalos amarillos con guía de polinización vino tinto, ovario blanco.

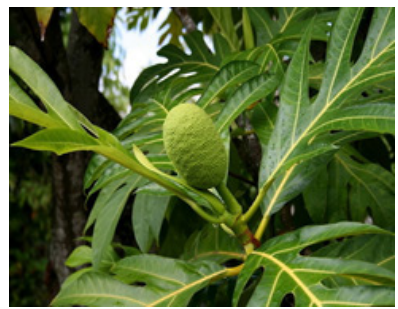

Figura 4. Árbol del pan (Artocarpus altilis). Árbol de hasta $20 \mathrm{~m}$ altura, tienen látex blanquecino. Presenta estípulas a manera de uña terminal en cada rama, deciduas, al caer dejan una cicatriz anular en cada nudo. Hojas generalmente lobuladas y miden hasta 70 $\mathrm{cm}$ largo y $50 \mathrm{~cm}$ ancho. Las flores masculinas están congregadas en espigas cilíndricas y de color amarillo; las flores femeninas se encuentran en capítulos. La infrutescencia mide hasta $40 \mathrm{~cm}$ largo y $20 \mathrm{~cm}$ ancho (González et al. 1995).

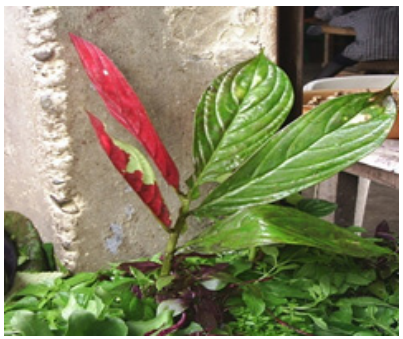

Figura 5. Riñonera (Columnea consanguínea). Hierba semi-epifita de tamaño de $50 \mathrm{~cm}$. Hojas simples, opuestas con una estípula foliar opuesta a la hoja, dos puntos rojos cerca del ápice principalmente en el envés. Tallo redondeado cubierto por brácteas. Flores axilares, cáliz amarillo, corola amarilla, brácteas rojas. Fruto pequeño, redondo, blanco (Pino-Benítez 2006). 


\section{Bioetnia Volumen 11, 2014}

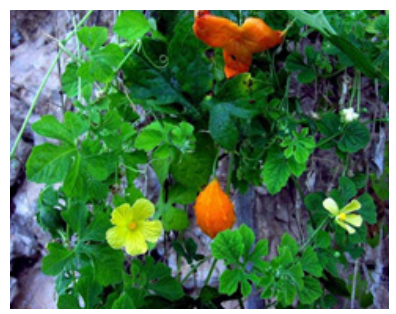

Figura 6. Balsamina (Momordica charantia). Es un bejuco herbáceo, trepador, anual, con zarcillos axilares simples, tallos con ángulos. Hojas cordiformes (en forma de corazón) son de color verde claro y pueden medir de 5 a $6 \mathrm{~cm}$ de largo y de 8 a $10 \mathrm{~cm}$ de ancho. Sus flores femeninas miden de 5 a $10 \mathrm{~cm}$ de largo y sus flores masculinas, más pequeñas son de color amarillo. Frutos tipo baya, oblongos a elipsoides, tuberculados, verdes, que varían a amarillo naranja al madurar, mide de 3 a $15 \mathrm{~cm}$ de largo, semillas con arilo rojo brillante, algo dulce (Bernal y Correa 1990).

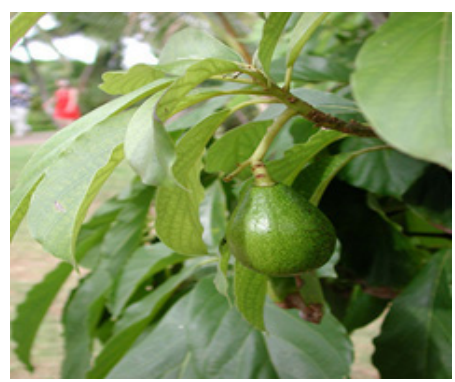

Figura 7. Aguacate (Persea americana Mill). Árbol de hasta $25 \mathrm{~m}$ de altura, tronco de hasta $80 \mathrm{~cm}$ de diámetro, erecto, con corteza escamosa y de colores grises. Hojas de $15 \mathrm{~cm}$ de longitud por $7 \mathrm{~cm}$ de ancho, simples, alternas, con borde entero, coriáceas, haz de color verde oscuro y brillante, envés de color blancuzco, forma elíptica, ápice terminado en punta aguda, con base cuneada y con nerviación marcada, sin estípulas. Flores amarillas verdosas, miden $1 \mathrm{~cm}$ de largo, tienen como característica que los androceos (órganos masculinos) maduran primero que los gineceos (órganos femeninos) con el propósito de impedir la autofertilización en la misma flor y dispuestas en racimos terminales erectos. Fruto de color verde, amarillo lustroso o púrpura con manchas de color gris (lenticelas), de 12 $\mathrm{cm}$ de diámetro que una semilla generalmente de forma alargada o redondeada. Semillas de color café, carnosas de forma redondeada y $5 \mathrm{~cm}$ de diámetro (Mahecha et al. 2004).

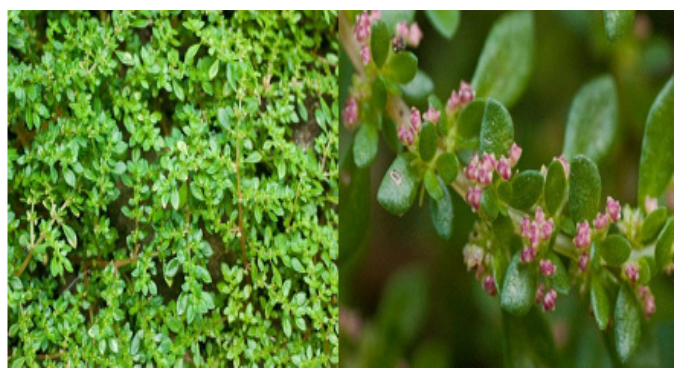

Figura 8. Mal de hígado (Pilea microphylla). Hierba que crece postrada o suberecta, carnosa. Hojas de forma obovada o elíptica, unos $10 \mathrm{~mm}$ de largo por $6 \mathrm{~mm}$ de ancho y margen entero. Flores de color verde amarillento. Frutos, aquenios de forma oblonga (Flores 2009 )..

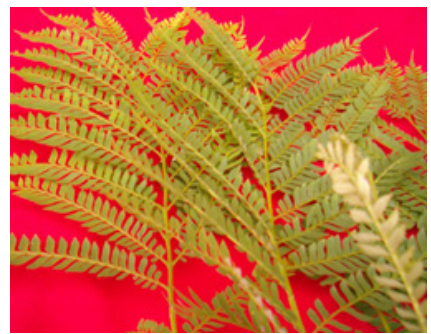

Figura 9. Pinguasí o gualanday (Jacaranda hesperia Dugand). Árbol de 7 a $10 \mathrm{~m}$ de alto; ramas lenmticelada, hojas dispuestas en espiral, imparipinnadas, bipinnadas, pinnas de $8-9 \mathrm{~cm}, 6$ en total, separadas $2 \mathrm{~cm}$ entre sí, raquis alado, folíolos ovado elípticos, $1.5 \mathrm{~cm}$, margen revoluta, base del pecíolo de loa hoja con lenticelas, base oblicua, ápice acuminado; nervio del folíolo impreso en el haz, prominente en el envés y este último de color plata, fruto, una cápsula plana oblongo-espiralada, con márgenes ondulados, ápice acuminado y base cuneadas; coriáceo-pétreo al secar; $15.5-17.5 \mathrm{~cm}$ de largo 8-9 cm de ancho. Semillas aladas. 


\section{Medicina tradicional y diabetes. CA Rentería et al.}

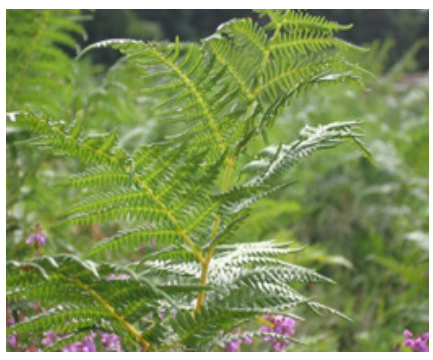

Figura 10. Helecho (Pteridium aquilinum). Helecho en forma de rizoma largo, reptante, cubierto densamente con pelos de color castaño claros a oscuros. Tiene un pecíolo fuerte, a veces acanalado de color castaño claro y sin pelos (glabro). Hojas pinnadas, de haz glabro y envés densamente cubierto con pelos septados y glandulares (Guzmán 2007).

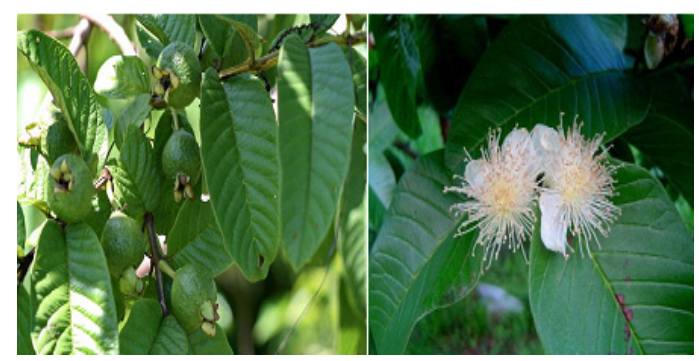

Figura 11. Guayaba (Psidium gaujava). Árbol o arbusto de hasta $10 \mathrm{~cm}$ de alto. Corteza lisa café clara con escamas que caen. Hojas elípticas, dos o tres veces más largas que anchas. Tienen fuerte olor al estrujarlas. Flores axilares, generalmente solitarias o en grupos de tres, blancas. Frutos redondos, carnosos, verduzcos, amarillos, hasta $6 \mathrm{~cm}$ de diámetro.

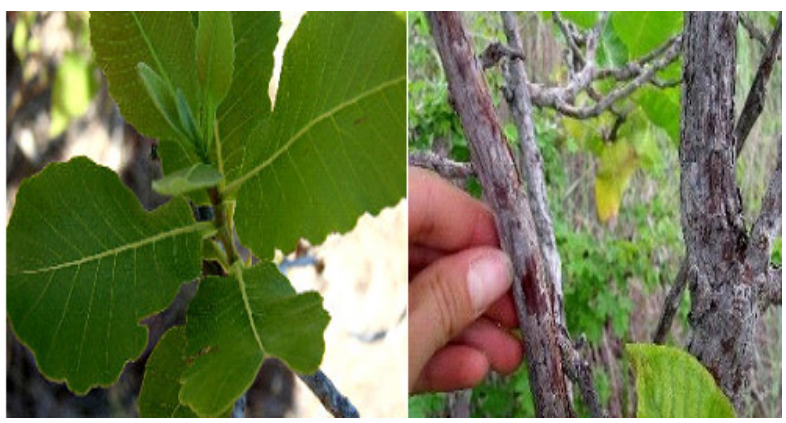

Figura 12. Chaparro rojo (Curatella americana). Arbolito de 4 a $6 \mathrm{~m}$ de alto. Con ramillas escamosas con numerosos nudos y entrenudos cortos. Hojas alternas, el haz y el envés foliar son ásperos al tacto, la nerviación es prominente por el envés, la lámina foliar es oblonga, el ápice redondeado, la base es angosta o truncada, de 6 a $16 \mathrm{~cm}$ de longitud y de 2 a $8 \mathrm{~cm}$ de ancho, con borde aserrado. Las inflorescencias son en racimos caulinares, de 2 a $3 \mathrm{~cm}$ pedicelados, con 7 a 14 flores. Los frutos cuando están inmaduros son globosos e híspidos (Barbosa-Castillo 1992).

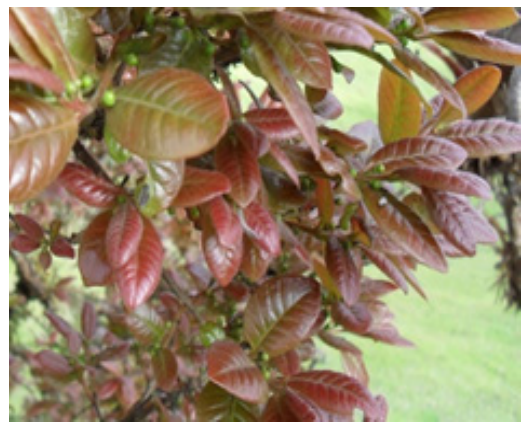

Figura 13. Arrayán (Myrtus foliosa). Árbol de copa con forma globosa, densa, con ramas tortuosas, angulosas y algunas veces nudosas. Es una planta perennifolia; el tallo presenta una corteza de color rojizo-parduzca que se descorteza en tiras o cueros pequeños; las hojas son opuestas, de textura coriácea, glabras de forma anchamente elíptica, resinosas, aromáticas, con nervaduras salientes en ambas caras pero más en la inferior. Las flores son de color blanco-amarillentas. El fruto es grande de forma globosa, verde cuando joven y rojo-pálido o amarillo crema al madurar, presentan una sola semilla de forma igualmente globosa (Rodríguez et al. 1984). 


\section{Bioetnia Volumen 11, 2014}

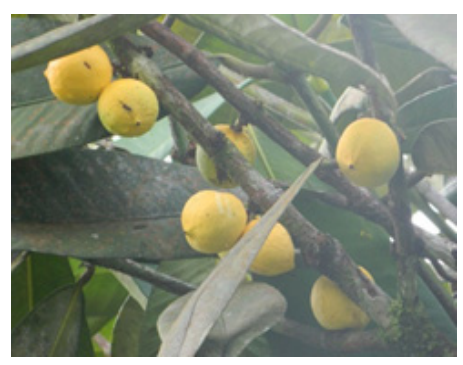

Figura 14. Madroño (Garcinia madruno). Árbol de 3 a $20 \mathrm{~m}$ de altura, tronco cilíndrico; corteza externa grisácea, con lenticelas dispuestas verticalmente; corteza interna gruesa, anaranjada; madera amarilla; látex amarillo o anaranjado, pegajoso y que fluye en puntos. Hojas simples, opuestas, decusadas, sin estipulas, $6-15 \mathrm{~cm}$ de largo y $2-9 \mathrm{~cm}$ de ancho, cartáceas, discoloras, verde oscuras y semilustrosas en la haz, verde claro en el envés, elípticas, ápice acuminado, base aguda; pecíolos de 1-2.5 cm de longitud; nerviación verde-amarillenta, un poco prominente. Flores en fascículos axilares, sésiles, 3 a 6 por axila; flores con pedicelos largos, 2.5-3.5 de longitud; sépalos 2, rojos, redondeados hacia el ápice, hasta $3 \mathrm{~mm}$ de longitud; pétalos 4 , amarillentos, orbiculares, hasta $7 \mathrm{~mm}$ de longitud. Frutos en baya, amarillos, verrugosos, 3-5 cm de largo y 3-3.5 de ancho; 2-3 semillas, más largas que anchas, marrón oscuro con arilo blanco.

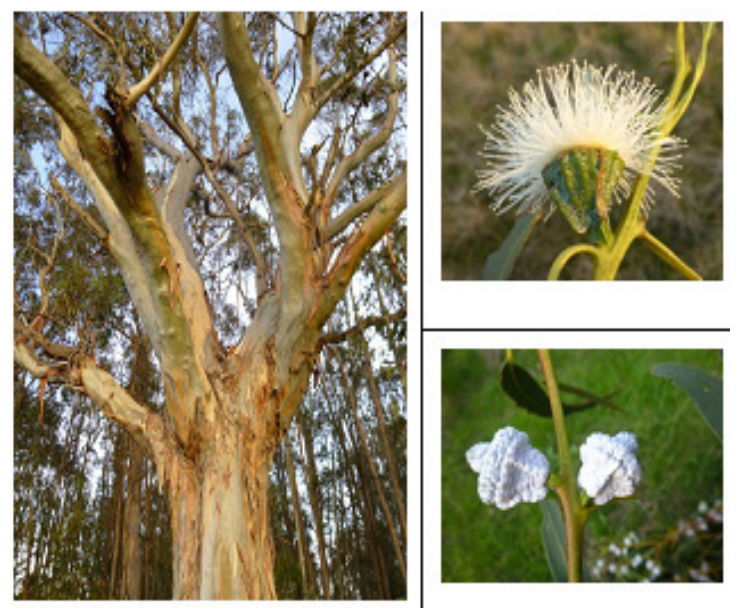

Figura 15. Eucalipto (Eucalyptus globulus). Árbol de hasta 45-75 m de altura. Tronco derecho que puede alcanzar los $2 \mathrm{~m}$ de diámetro y tendente a la torsión helicoidal, tiene la corteza caediza, que solo aguanta en la base del mismo, es lisa, fibrosa, al principio blanco cremosa y que luego amarilla grisácea o parduzca con tonos azulados. Las hojas son dimórficas, las juveniles de 4-16 x 1,5-9 $\mathrm{n} \mathrm{cm}$, son opuestas, ovadas u ovados lanceoladas, con base redondeada, falciformes, con base atenuada, el ápice agudo y un nervio central marcado. Las flores se reúnen en inflorescencia tipo umbela en las que hay 1 (generalmente), 3 o 7 flores, estas nacen de botones cuadrangulares de 20 a 30 turbinados y glaucos. El cáliz está formado por 4 sépalos, la corola por 4 pétalos; el androceo tiene numerosos estambres, con filamentos filiformes y libres (Menéndez et al. 2004).

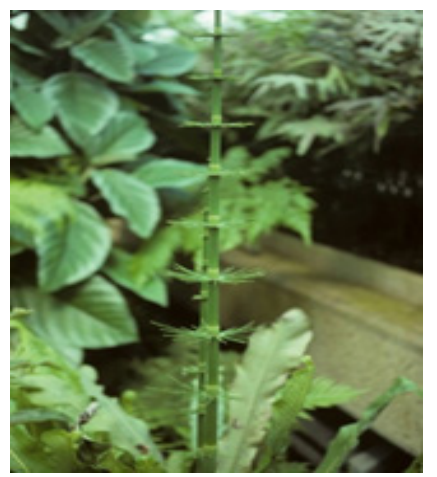

Figura 16. Cola de caballo (Equisetum giganteum). Subarbusto que en estado juvenil tiene tallos erectos, que al madurar se tornan semitrepadores, alcanzan los dos metros o más de altura, gruesos, con crestas y valles. Hojas modificadas a manera de escamas (escuamiformes), frágiles, caedizas; ramas densamente verticiladas, textura áspera. Tallos fértiles similares a los estériles, los fértiles con estróbilos apicales. Reproducción por esporas (Mahecha et al. 2004). 


\section{Medicina tradicional y diabetes. CA Rentería et al.}

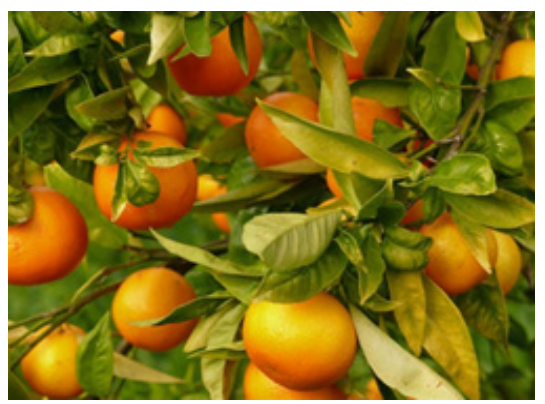

Figura 17. Mandarina (Citrus nobilis). Árbol pequeño de 2-6 m de altura, con tronco con frecuencia torcido, generalmente sin espinas. Ramillas angulosas. Hojas oblongo-ovales, elípticas o lanceoladas, de $3.5-8 \mathrm{~cm}$ de longitud y $1.5-4 \mathrm{~cm}$ de anchura, con la base y el ápice obtusos. Margen aserrado por encima de la base. Son de color verde oscuro brillante en el haz y verde amarillento en el envés, fragantes cuando se las tritura. Pecíolos con ala muy corta. Inflorescencias axilares o terminales con 1-4 flores pentámeras, de color blanco, olorosas, de 1.5-2.5 cm de diámetro. 18-23 estambres, casi libres. Frutos de 4-7 cm de longitud y 5-8 cm de diámetro, globosodeprimidos. Su color varía de amarillo verdoso al naranja y rojo anaranjado. La superficie es brillante y está llena de glándulas oleosas hundidas. La cáscara es delgada, muy fragante, separándose fácilmente de la pulpa. Pulpa jugosa y dulce, refrescante. Semillas oblongo-ovoides.

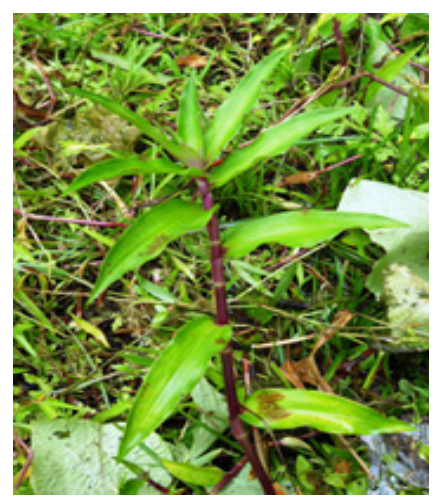

Figura 18. Siempreviva o Chicoria (Tripogandra serrulata). Hierba perenne, de raíz fibrosa con adventicias en los nudos. Tallo procumbente, de color verde rayado de purpura glabro y suculento. Hojas alterna, simples, carnosas, verde pálido en el envés, lanceoladas, sésiles, glabras. Vaina glabra, puberulenta a lo largo de uno de los lados. Flores con sépalos verde pálido, piloso, pétalos blancos, se agrupan en una umbela, fruto capsula con tres celdas.
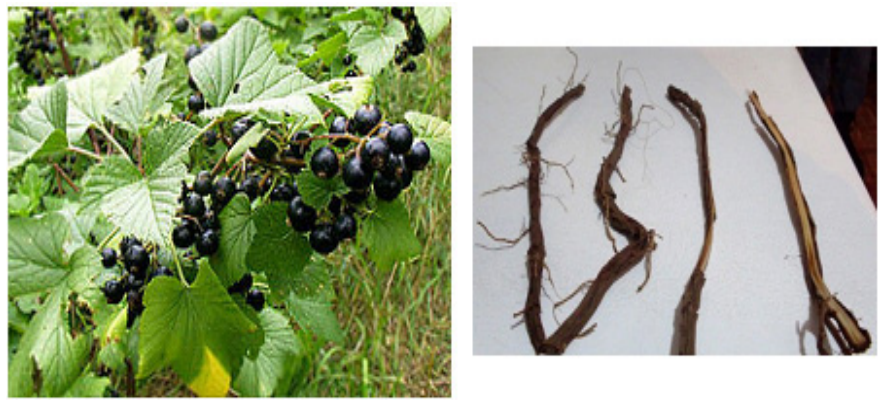

Figura 19. Zarzaparrilla (Smilax ornata Lem). Es un arbusto con los tallos delgados, volubles, de uno a dos metros de largo y espinosas, hojas pecioladas, alternas, ásperas, con muchos nervios, acorazonadas, y persistentes, las flores de color amarillo-crema en racimos axilares, los frutos en forma de bayas globosas como el guisante y raíces fibrosas y casi cilíndricas utilizadas en medicinas en medicamentos sudoríficos y depurativos. El fruto contiene de una a tres semillas. En la base del pecíolo de cada hoja, hay dos zarcillos. El fruto no se utiliza, lo que se utiliza es su raíz. Su fruto contiene 6 semillas y no tiene zarcillos. 